\title{
DÉTERMINATION DE LA PERMÉABILITÉ COMPLEXE EN RÉSONANCE FERROMAGNÉTIQUE
}

\author{
par R. LANCIA et A. MARCHAND \\ Laboratoire d'Electrostatique et de Physique du Métal, C. N. R. S., Grenoble, \\ Faculté des Sciences de Grenoble
}

(Reçu le 3 juin 1970)

\begin{abstract}
Résumé. - Les auteurs présentent une méthode de mesure qui permet la conversion des résultats expérimentaux (courant détecté) en valeurs non arbitraires de chacune des deux parties, réelle et imaginaire, de la perméabilité complexe.

L'utilisation d'un abaque rend cette transformation particulièrement simple.
\end{abstract}

\begin{abstract}
A new method of measurement is presented which permits the transformation of the experimental results in non-arbitrary values of both the real and imaginary parts of the complex permeability. In particular the utilization of a chart makes very easy the transformation.
\end{abstract}

Introduction. - Nous avons étudié par résonance magnétique, à $9,2 \mathrm{GHz}$, des composés intermétalliques de cobalt et de terre rare. Suivant la terre rare considérée et la température de l'échantillon, ces composés sont soit para-, soit ferro-, soit ferrimagnétiques. Les résultats de ces études ont été publiés $[1,2,3]$.

Nous exposons ci-après la méthode utilisée pour obtenir les courbes de résonance $\mu_{\mathrm{R}}(H)$ (absorption effective) à partir des résultats expérimentaux. Ces derniers se présentent sous forme de courbes obtenues par enregistrement direct du courant fourni par un détecteur à cristal (courant proportionnel à la puissance réfléchie par la cavité hyperfréquence au sein de laquelle est placé l'échantillon du composé étudié).

Méthode de mesure. - La méthode, très classique, que nous avons adoptée, est fondée sur l'étude des perturbations des caractéristiques d'une cavité résonnante hyperfréquence résultant de la présence, au sein de la cavité, d'un échantillon du matériau magnétique à étudier.

Dans les expériences de résonance sur les matériaux magnétiques isolants, la partie réelle $\mu^{\prime}$ de la perméabilité réversible complexe $\left(\mu=\mu^{\prime}-j \mu^{\prime \prime}\right)$ est déterminée à partir de la modification de la fréquence de résonance de la cavité de mesure (dispersion) et la partie imaginaire $\mu^{\prime \prime}$ à partir de la variation du facteur de surtension de la cavité (absorption).

Dans le cas d'un matériau magnétique conducteur, les pertes électriques importantes causées par la résistance finie du matériau ne peuvent pas être séparées physiquement des pertes magnétiques. L'influence de l'épaisseur de la couche de peau dans l'échantillon se manifeste à la fois sur les pertes et sur la fréquence de résonance de la cavité.
Si l'échantillon constitue une paroi ou partie d'une paroi plane de la cavité, d'une part, si la direction du champ magnétique statique, perpendiculaire à celle du champ magnétique hyperfréquence, est parallèle à cette paroi [4], d'autre part, on trouve [5], dans une approximation du premier ordre en $1 / Q$ :

$$
\begin{gathered}
\frac{1}{Q_{\mathrm{fer}}}=\mu_{\mathrm{R}}^{1 / 2}-\frac{1}{Q_{0}} \\
-\frac{2 \Delta \omega}{\omega_{0}}= \pm \mu_{\mathrm{L}}^{1 / 2}-\frac{1}{Q_{0}}\left\{\begin{array}{ccc}
+ & \text { si } & \mu^{\prime}>0 \\
- & \text { si } & \mu^{\prime}<0
\end{array}\right.
\end{gathered}
$$

avec

$$
\begin{aligned}
& \mu_{\mathrm{R}}=\left(\mu^{\prime 2}+\mu^{\prime \prime 2}\right)^{1 / 2}+\mu^{\prime \prime} \\
& \mu_{\mathrm{L}}=\left(\mu^{\prime 2}+\mu^{\prime \prime 2}\right)^{1 / 2}-\mu^{\prime \prime}
\end{aligned}
$$

(on remarque :

$$
\begin{gathered}
\left.\left[2 j\left(\mu^{\prime}-j \mu^{\prime \prime}\right)\right]^{1 / 2}=\mu_{\mathrm{R}}^{1 / 2}+j \mu_{\mathrm{L}}^{1 / 2}\right) \\
Q_{0}=\frac{2}{\delta_{0}} \frac{\iiint H^{2} \mathrm{~d} V}{\iint H^{2} \mathrm{~d} S} .
\end{gathered}
$$

Dans cette dernière expression, où l'intégrale de surface est étendue à la surface de l'échantillon magnétique seulement et l'intégrale triple au volume interne de la cavité, $Q_{0}$ représente le facteur de surtension associé aux pertes dans l'échantillon dans le cas particulier où la perméabilité de celui-ci est réputée égale à l'unité ; $\delta_{0}$ est l'épaisseur de la couche de peau $(\mu=1)$ et $H$ le champ magnétique hyperfréquence. 
$Q_{\text {fer }}$ représente le facteur de surtension associé à l'ensemble des pertes dans l'échantillon et $\omega_{0}$ la pulsation de résonance de la cavité.

Ainsi, avec un matériau magnétique conducteur, au lieu de mesurer directement $\mu^{\prime}$ et $\mu^{\prime \prime}$ comme dans le cas d'un matériau isolant, on mesure $\mu_{\mathbb{R}}$, absorption effective et $\mu_{L}$, dispersion effective; $\mu_{\mathrm{R}}$ et $\mu_{L}$ étant connus, nous pouvons définir $\mu^{\prime}$ et $\mu^{\prime \prime}$ :

$$
\mu^{\prime 2}=\mu_{\mathrm{R}} \mu_{\mathrm{L}} ; \quad \mu^{\prime \prime}=\frac{\mu_{\mathrm{R}}-\mu_{\mathrm{L}}}{2} .
$$

Les composés magnétiques que nous étudions sont conducteurs. Ils nous ont été fournis sous forme de poudres dont les grains ont un diamètre de 20 microns environ. La pénétration du champ hyperfréquence (épaisseur de la couche de peau) à l'intérieur des grains, aux fréquences voisines de $10 \mathrm{GHz}$, est de l'ordre de 2 à 3 microns.

Il était très intéressant de pouvoir expliciter nos expériences à l'aide des équations simples précédentes. Cela n'était possible que si l'échantillon se présentait sous une forme qui permit de l'assimiler à un élément de paroi de la cavité. Cette condition fut satisfaite en réalisant l'échantillon par dépôt, sur le fond de la cavité de mesure (en sa région centrale où l'intensité du champ magnétique hyperfréquence est maximale), d'une mince couche d'un mélange de la poudre à étudier avec un liant. Le liant que nous avons adopté, après divers essais, est une graisse aux silicones; cette graisse, dont les propriétés demeurent inaltérées aux températures les plus élevées de nos expériences, protège les grains magnétiques de l'oxydation lors des montées en température.

Lorsque les phénomènes de résonance sont de faible intensité et sont, de ce fait, plus ou moins masqués par le bruit de fond, la méthode très couramment mise en œuvre pour améliorer le rapport signal/bruit consiste à faire usage d'un détecteur synchrone (ce qui implique que l'on module le champ magnétisant). Les courbes traduisant l'évolution, en fonction du champ, du signal délivré par le détecteur (courbes généralement obtenues à l'aide d'un enregistreur) constituent une représentation première de la dérivée, par rapport au champ, de l'absorption.

Cette particularité n'est pas gênante car, de ces courbes, on peut déduire sans spéciales difficultés les caractéristiques les plus significatives du phénomène de résonance, à savoir : les valeurs des champs de résonance, d'une part, les largeurs des courbes d'absorption (largeurs à mi-hauteur), d'autre part.

Le phénomène de résonance dans les composés que nous étudions, en bande $X$, s'est toujours, sauf dans le cas de $\mathrm{Tb}_{3} \mathrm{Co}$, manifesté de manière très nette. Les variations du signal issu du cristal détecteur de mesure furent toujours de bonne amplitude comparativement au niveau du bruit de fond. Dans ces conditions, nous avons pu effectuer nos expériences à l'aide d'un appareillage relativement simple.
Nous précisons que notre cavité de mesure est « montée en réflexion » et que le cristal détecteur de mesure délivre un courant proportionnel à la puissance réfléchie par la cavité (fonctionnement du cristal en régime quadratique vérifié à partir de tests effectués à l'aide d'un affaiblisseur hyperfréquence étalonné).

Les courbes $i_{\mathrm{r}}(H)$, que nous obtenons directement par enregistrement, représentent l'évolution du courant détecté, à puissance hyperfréquence incidente constante, en fonction du champ magnétique dans l'entrefer de l'électro-aimant (entrefer au sein duquel est placée la cavité). De ces courbes, qui nous fournissent une première vision du phénomène d'absorption, il faut déduire celles représentatives de $\mu_{\mathrm{R}}(H)$. Pour cela, il est nécessaire d'effectuer des mesures auxiliaires de facteurs de surtension caractéristiques de la cavité de mesure.

Détermination de $\mu_{\mathbf{R}} \cdot-$ Considérons deux points de la courbe $i_{\mathrm{r}}(H)$ correspondant aux champs magnétiques $H_{1}$ et $H_{2}$. Désignons par $\mu_{\mathrm{R}_{1}}$ et $\mu_{\mathrm{R}_{2}}, \beta_{1}$ et $\beta_{2}$, les valeurs de l'absorption effective et du coefficient de couplage de la cavité au guide d'onde qui correspondent à $H_{1}$ et $H_{2}$ respectivement.

A partir de l'équation (1) et des définitions des facteurs de surtension et du coefficient de couplage d'une cavité (se reporter aux annexes), on peut écrire :

$$
\begin{aligned}
& \frac{1}{\beta_{1}}=Q_{\mathrm{e}}\left(\frac{1}{Q_{\mathrm{c}}}+\frac{1}{Q_{0}} \mu_{\mathrm{R}_{1}}^{1 / 2}\right) \\
& \frac{1}{\beta_{2}}=Q_{\mathrm{e}}\left(\frac{1}{Q_{\mathrm{c}}}+\frac{1}{Q_{0}} \mu_{\mathrm{R}_{2}}^{1 / 2}\right) .
\end{aligned}
$$

Dans ces expressions, $Q_{\mathrm{e}}$ est le facteur de surtension externe de la cavité, $Q_{\mathrm{c}}$ celui associé aux pertes dans la paroi interne de la cavité (la surface à considérer est celle de la paroi diminuée de la surface occupée par l'échantillon); la définition du facteur de surtension $Q_{0}$ a été donnée précédemment.

Des deux relations qui précèdent, on tire la suivante :

$$
\mu_{R_{1}}^{1 / 2}=\mu_{R_{2}}^{1 / 2}+\left(\frac{1}{\beta_{1}}-\frac{1}{\beta_{2}}\right) \frac{Q_{0}}{Q_{\mathrm{e}}} .
$$

Cette relation permet le calcul de $\mu_{\mathrm{R}_{1}}$ à partir d'une valeur de référence : $\mu_{\mathrm{R}_{2}}$ correspondant à un champ $\mathrm{H}_{2}$.

A l'instar de divers auteurs, nous mettrons à profit le fait que $\mu_{\mathrm{R}}$ peut être assimilé à l'unité aux champs très élevés $[5,6]$ (sinon très élevés du moins grands relativement au champ de résonance). Cette assimilation pouvait être faite dans le cadre de nos expériences.

Désignant par $\beta_{0}$ le coefficient de couplage de la cavité sous champ élevé, l'expression (8) prend la forme particulière suivante :

$$
\mu_{\mathrm{R}_{1}}^{1 / 2}=1+\left(\frac{1}{\beta_{1}}-\frac{1}{\beta_{0}}\right) \frac{Q_{0}}{Q_{\mathrm{e}}} .
$$


Passage de la courbure enregistrée $i_{\mathrm{r}}(H)$ à celle d'absorption effective $\boldsymbol{\mu}_{\mathrm{R}}(\boldsymbol{H})$. - Nous exploiterons l'équation (9). Dans cette équation, $\beta_{0}$ et $\beta_{1}$ sont les coefficients de couplage de la cavité sous champ élevé et sous champ $H_{1}$ respectivement.

Nous obtenons, compte tenu du lien entre coefficients de couplage $\beta$ et de réflexion $\mathcal{R}$ dans le cas du souscouplage (voir plus loin « Remarque 2 ») :

$$
\begin{aligned}
\mu_{R_{1}}^{1 / 2} & -1=\frac{2 Q_{0}}{Q_{\mathrm{e}}} \times \\
& \times \frac{\left|\mathcal{R}_{1}\right|^{2}-\left|\mathcal{R}_{0}\right|^{2}}{\left(\left|\mathcal{R}_{1}\right|+\left|\mathcal{R}_{0}\right|\right)\left(1-\left|\mathcal{R}_{1}\right|\right)\left(1-\left|\mathcal{R}_{0}\right|\right)} .
\end{aligned}
$$

Par définition, $|\mathcal{R}|^{2}=P_{\mathrm{r}} / P_{\mathrm{i}} \quad$ expression dans laquelle $P_{\mathrm{r}}$ et $P_{\mathrm{i}}$ sont les puissances réfléchie et incidente respectivement. Nous aurons :

$$
\left|\mathcal{R}_{1}\right|^{2}-\left|\mathcal{R}_{0}\right|^{2}=\frac{P_{\mathrm{r}_{1}}}{P_{\mathrm{i}_{1}}}-\frac{P_{\mathrm{r}_{0}}}{P_{\mathrm{i}_{0}}}=\frac{P_{\mathrm{r}_{1}}-P_{\mathrm{r}_{0}}}{P_{\mathrm{i}_{1}}}
$$

( $P_{\mathrm{i}}$ est maintenue constante).

Notre cristal détecteur d'onde réfléchie fonctionnant en régime quadratique, le courant qu'il délivre est proportionnel à la puissance réfléchie.

Désignons par $i_{\mathrm{r}_{1}}$ le courant détecté correspondant à la puissance réfléchie par la cavité sous champ $H_{1}$, par $i_{\mathrm{r}_{0}}$ celui obtenu lorsque la cavité est sous champ élevé et par $i_{1}$ celui correspondant à la puissance incidente (ce dernier courant est obtenu en substituant un court-circuit à la cavité).

Nous pouvons écrire :

$$
\left|\mathcal{R}_{1}\right|^{2}-\left|\mathcal{R}_{0}\right|^{2}=\left(i_{\mathrm{r}_{1}}-i_{\mathrm{r}_{0}}\right) / i_{1} .
$$

L'équation (10) peut alors être mise sous la forme suivante :

$$
\mu_{\mathrm{R}_{1}}^{1 / 2}-1=\frac{2 Q_{0}}{Q_{\mathrm{e}}} \frac{i_{\mathrm{r}_{1}}-i_{\mathrm{r}_{0}}}{i_{1}} \frac{1}{D_{01}} .
$$

Dans cette équation, $D_{01}$ représente le terme dénominateur :

$$
\left(\left|\mathcal{R}_{1}\right|+\left|\mathcal{R}_{0}\right|\right)\left(1-\left|\mathcal{R}_{1}\right|\right)\left(1-\left|\mathcal{R}_{0}\right|\right) .
$$

$\mathrm{Au}$ cours d'une expérience de résonance, seuls $i_{\mathrm{r}_{1}}$ et $\mathcal{R}_{1}$ varient avec le champ $H$. Nous pouvons mettre à profit ce fait pour présenter l'équation (11) sous une forme plus utilisable, à savoir :

$$
\mu_{\mathrm{R}_{1}}^{1 / 2}-1=\frac{A}{D_{01}} \frac{i_{\mathrm{r}_{1}}-i_{\mathrm{r}_{0}}}{i_{\mathrm{r}_{0}}} .
$$

Dans cette équation, $A=\left(2 Q_{0} / Q_{\mathrm{e}}\right)\left(i_{\mathrm{r}_{0}} / i_{1}\right)$ et $i_{\mathrm{r}_{0}}$ sont des termes qui demeurent constants au cours d'une expérience; $A$ peut donc être calculé dès achèvement des mesures de surtensions.

Quant au facteur $D_{01}$, celui-ci peut être tiré d'un abaque établi en prenant pour paramètre $\left|\mathcal{R}_{0}\right|$ (indépendant de $H$ ) et $\left|\mathcal{R}_{1}\right|$ pour variable d'entrée. Nous avons établi un tel abaque esquissé figure 1.

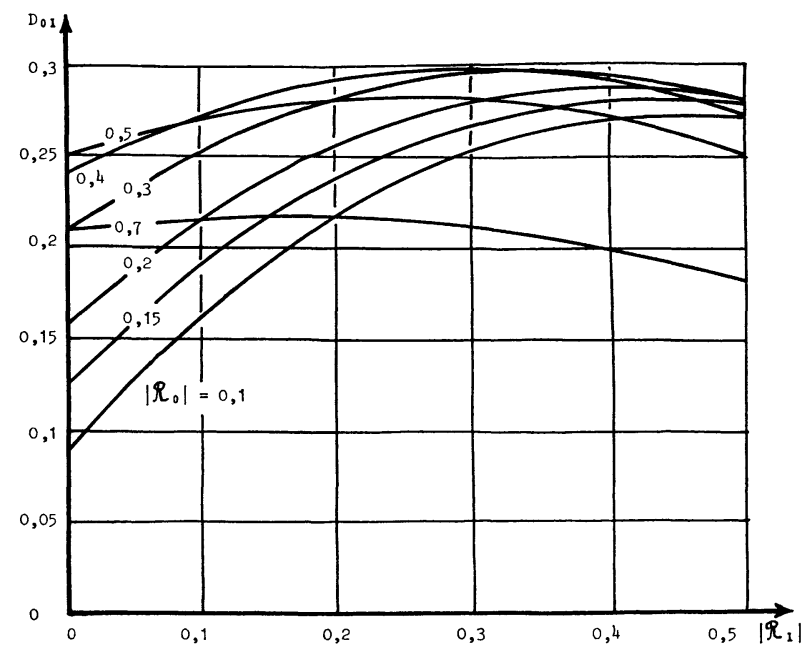

FIG. 1.

Afin de faciliter les opérations graphiques, on aura intérêt à tracer d'emblée, à partir de la courbe $i_{\mathrm{r}}(H)$, la courbe $y(H)=\left(i_{\mathrm{r}} / i_{\mathrm{r}_{0}}\right)(H)$.

Le calcul de $\left|\mathcal{R}_{1}\right|$, en particulier, sera facilité ; en effet, nous aurons simplement:

$$
\left|\mathcal{R}_{1}\right|=\sqrt{\left(i_{\mathrm{r}_{1}} / i_{1}\right)}=\left|\mathcal{R}_{0}\right| \sqrt{\left(i_{\mathrm{r}_{1}} / i_{\mathrm{r}_{0}}\right)}=\left|\mathcal{R}_{0}\right| \sqrt{y_{1}} .
$$

Par ailleurs, nous aurons :

$$
\mu_{\mathrm{R}_{1}}^{1 / 2}-1=\frac{A}{D_{01}}\left(y_{1}-1\right) .
$$

La figure 2 résume les diverses opérations à effectuer pour passer de $i_{\mathrm{r}}(H)$ à $\mu_{\mathrm{R}_{1}}^{1 / 2}(H)$ :

a) calculs initiaux de $A$ et $\left|\mathcal{R}_{0}\right|$;

b) pour $H_{1}$, calculer $\left|\mathcal{R}_{1}\right|=\left|\mathcal{R}_{0}\right| y_{1}^{1 / 2}$ et tirer $D_{0_{1}}$ de l'abaque (Fig. 1);

c) calculer $\mu_{\mathrm{R}}^{1 / 2}-1=\left(A / D_{01}\right)\left(y_{1}-1\right)$; soit :

$$
E C=\left(\frac{A}{D_{01}}\right) E B \text {. }
$$

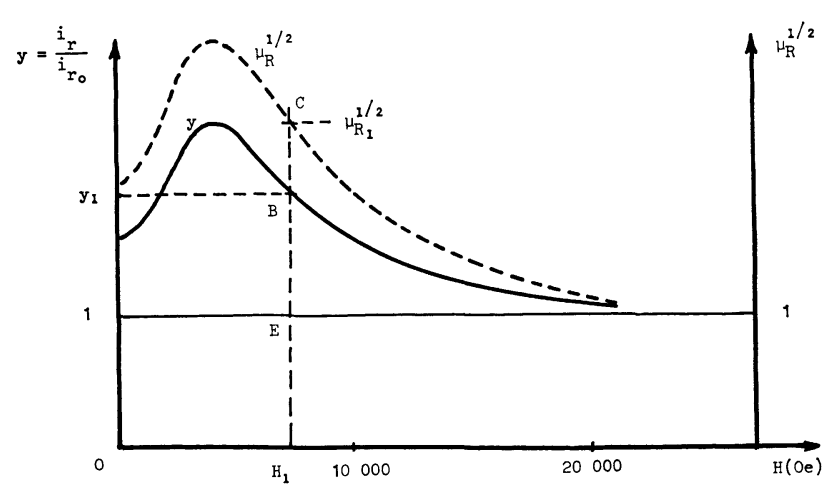

FIG. 2.

Remarque 1. - La relation $\left(12^{\prime}\right)$ suggère la remarque suivante : si $D_{01}$ varie très peu dans tout le domaine de variation de $H$, alors $\left(\mu_{\mathrm{R}}^{1 / 2}-1\right)$ s'identifie à $(y-1)$ (au facteur multiplicatif $A / D_{01}$ près). 
Un examen de l'abaque révèle qu'une telle situation peut se rencontrer si $\left|\mathcal{R}_{0}\right|$ est de l'ordre de 0,$3 ;\left|\mathcal{R}_{1}\right|$ minimum étant égal à $\left|\mathcal{R}_{0}\right|, D_{01}$ varie en effet de moins de $6 \%$ lorsque $\left|\mathcal{R}_{1}\right|$ varie entre 0,3 et 0,5 . Il faut noter qu'une valeur de $\left|\mathcal{R}_{0}\right|$ de l'ordre de 0,3 est assez élevée et qu'elle ne peut être obtenue qu'au prix d'un amortissement relativement important de la cavité par l'échantillon.

REMARQue 2. - Le procédé précédemment développé n'est applicable qu'au cas d'une cavité demeurant constamment sous-couplée. On peut évidemment le transposer au cas du sur-couplage mais il faut noter que l'emploi d'une cavité sur-couplée peut présenter des inconvénients en ce sens que, le coefficient de couplage diminuant lorsque les pertes augmentent, une telle cavité peut se trouver dans des états de souscouplage en cours d'expérience.

Détermination de $\mu_{\mathbf{L}}$. - Il est nécessaire, si l'on veut connaître $\mu^{\prime \prime}$, comme $\mu^{\prime}$ d'ailleurs, de mesurer à la fois $\mu_{\mathrm{R}}$ et $\mu_{\mathrm{L}}$.

La dispersion est définie par l'équation (2) que nous rappelons : $-2\left(\Delta \omega / \omega_{0}\right)= \pm \mu_{\mathrm{L}}^{1 / 2}\left(1 / Q_{0}\right) \quad$ (signe $+\mathrm{si}$ $\mu_{1}>0$, signe - si $\mu_{1}<0$; signe + dans le cas de nos expériences).

La valeur de $\mu_{\mathrm{L}}$ découle donc directement d'une mesure de la variation relative de la fréquence de résonance de la cavité. Au maximum d'absorption (résonance), la dispersion est nulle ; cet état constitue une référence.

Si la mesure de $\mu_{\mathrm{R}}$ ne présente en général pas de réelles difficultés expérimentales, par contre, celle de $\mu_{\mathrm{L}}$ est souvent très délicate en raison de la faible grandeur des variations relatives de la fréquence de résonance de la cavité.

De ce fait, l'obtention de résultats corrects lors de l'étude de la dispersion est subordonnée à l'emploi d'un spectromètre hyperfréquence sinon très perfectionné (voir par exemple [7]), du moins spécialement adapté à la détection de très faibles variations de fréquence.

Quoique simple, l'appareillage hyperfréquence que nous utilisons pour nos expériences dans la bande $3 \mathrm{~cm}$ permet, outre la détermination de $\mu_{\mathrm{R}}$, celle de la dispersion effective $\mu_{\mathbf{L}}$.

Dans notre installation, la fréquence des oscillations du klystron est en permanence ajustée sur celle de la cavité de mesure par réglage automatique de la tension réflecteur du klystron (ce point a été développé par ailleurs [3]).

Dans un petit domaine, au centre du mode selon lequel fonctionne le klystron, les variations de sa fréquence sont proportionnelles aux variations de la tension réflecteur. C'est de l'enregistrement de ces dernières au cours d'un cycle de variation du champ magnétisant que l'on déduira, sans difficultés, la courbe de dispersion effective $\mu_{\mathbf{L}}(H)$.

\section{Annexe 1. Facteurs de surtension.}

$Q_{\mathrm{e}}$ : facteur de surtension externe de la cavité ;

$Q_{\mathrm{i}}$ : facteur de surtension interne;

$Q_{\mathrm{L}}$ : facteur de surtension apparent (ou en charge) :

$$
1 / Q_{\mathrm{L}}=\left(1 / Q_{\mathrm{i}}\right)+\left(1 / Q_{\mathrm{e}}\right) ;
$$

$Q_{\mathrm{c}}$ : facteur de surtension associé aux pertes dans la paroi interne de la cavité (la surface à considérer est celle de la paroi diminuée de la surface occupée par l'échantillon);

$Q_{\mathrm{fer}}$ : facteur de surtension associé à l'ensemble des pertes dans l'échantillon:

$$
1 / Q_{\mathrm{i}}=\left(1 / Q_{\mathrm{c}}\right)+\left(1 / Q_{\mathrm{fer}}\right) ;
$$

$\beta \quad$ : coefficient de couplage de la cavité au guide d'ondes : $\beta=Q_{\mathrm{i}} / Q_{\mathrm{e}}$

$$
\begin{aligned}
& \beta<1 \text {, sous-couplage, } \\
& \beta>1 \text {, sur-couplage. }
\end{aligned}
$$

Annexe 2. Mesure de la surtension interne d'une cavité. - Selon le procédé que nous utilisons, décrit par Strub [8], la mesure de la surtension interne $Q_{\mathrm{i}}$ est fondée sur la mesure du coefficient de couplage, $\beta$, d'une part, sur la mesure, par déréglage de fréquence, de la surtension en charge $Q_{\mathrm{L}}$ de la cavité, d'autre part.

On a en effet :

$$
Q_{\mathrm{i}}=(1+\beta) Q_{\mathrm{L}}=(1+\beta) \frac{f_{0}}{2 \Delta f} .
$$

Dans cette relation, $f_{0}$ est la fréquence de résonance de la cavité et $2 \Delta f$, la largeur de la courbe de résonance à demi-puissance réfléchie. Dans le cas, qui est le nôtre, où le cristal détecteur de l'onde réfléchie fonctionne en régime quadratique, le courant qu'il délivre est proportionnel à la puissance réfléchie.

Soit $i_{1}$ le courant détecté lorsque la cavité est très désaccordée ou, mieux, lorsqu'on lui substitue un court-circuit. On a : $i_{1}=k|\mathcal{R}|^{2}=k(\mathcal{R}=1)$. Soit, d'autre part, $i_{\mathrm{r}}$ le courant détecté lorsque la cavité est à la résonance : $i_{\mathrm{r}}=k\left|\mathcal{R}_{\mathrm{r}}\right|^{2}=i_{1}\left|\mathcal{R}_{\mathrm{r}}\right|^{2}$.

Par suite :

$$
\left|\mathcal{R}_{\mathrm{r}}\right|=\sqrt{i_{\mathrm{r}} / i_{1}} \text { et } \rho_{\mathrm{r}}=\frac{1+\left|\mathcal{R}_{\mathrm{r}}\right|}{1-\left|\mathcal{R}_{\mathrm{r}}\right|} .
$$

On aura $: \beta=\rho_{\mathrm{r}}$ si $\beta>1$ (sur-couplage) $; \beta=1 / \rho_{\mathrm{r}}$ si $\beta<1$ (sous-couplage).

Nous déterminons $Q_{\mathrm{L}}$ par déréglage de la fréquence du klystron.

Il s'agit de définir expérimentalement la variation $2 \Delta f$ de la fréquence du klystron qui permet de passer d'un flanc à l'autre de la courbe de résonance de la cavité, en des points pour lesquels le détecteur délivre un même courant : $i^{\prime}=\left(i_{1}+i_{\mathrm{r}}\right) / 2$ (demi-puissance réfléchie).

La surtension en charge de la cavité est alors exprimée simplement : $Q_{\mathrm{L}}=f_{0} / 2 \Delta f$.

Parmi les divers procédés permettant la détermination de $2 \Delta f$, celui que nous avons employé consiste à 
modifier la fréquence du klystron par variation de sa tension réflecteur $V_{\mathrm{r}}$ (au centre d'un mode, $f$ varie linéairement avec $V_{\mathrm{r}}$ ).

Si $v$ est la variation de tension réflecteur correspondant à $2 \Delta f$, on a : $2 \Delta f=v\left(\Delta f / \Delta V_{r}\right)=v \varphi(\varphi$ est déterminé expérimentalement à l'aide d'un étalon de fréquence).

Pendant toute la durée de ces diverses mesures, il faut veiller à maintenir constante la puissance hyperfréquence incidente.

On notera que la méthode simple qui vient d'être décrite n'est pas facilement applicable à la mesure des surtensions très élevées, supérieures à 10000 par exemple.

APPliCATIONS A nOS EXPÉRIENCES. - Pour déterminer $\mu_{\mathrm{R}}$ après relevé d'une courbe $i_{\mathrm{r}}(H)$, on appliquera la relation (9) (ou toute autre équivalente) laquelle nécessite les déterminations de : $Q_{\mathrm{e}}$ : fonction seulement de la cavité ; $Q_{0}$ et $\beta_{0}$ : fonctions de l'échantillon; $\beta_{1}$.

$Q_{\mathrm{e}}$ sera déterminé à partir d'une mesure de surtension interne, mesure qui nécessite celle de $\beta$. On aura :

$$
Q_{\mathrm{e}}=Q_{\mathrm{i}} / \beta \text {. }
$$

DÉTERMINATION DE $Q_{0} \cdot-Q_{0}$ peut être calculé lorsque l'échantillon est homogène et que ses caractéristiques physiques sont bien connues [5]. Cela n'étant pas notre cas, nous déterminerons $Q_{0}$ à partir des mesures de la surtension interne $Q_{\mathrm{i}_{0}}$ sous champ élevé, de la cavité contenant l'échantillon et de la surtension $Q_{\text {c }}$.

On a en effet, sous champ élevé :

$$
1 / Q_{\mathrm{i}_{0}}=\left(1 / Q_{0}\right)+\left(1 / Q_{\mathrm{c}}\right) .
$$

La mesure de $Q_{\mathrm{i}_{0}}$ sera effectuée comme il a été montré.

Quant à la valeur de $Q_{\mathrm{c}}$, elle résultera d'une mesure de la surtension interne $Q_{\mathbf{i}_{\mathrm{c}}}$ de la cavité vide et du calcul d'un facteur correctif $\alpha$ dépendant de la surface occupée par l'échantillon au sein de la cavité.

La surtension $Q_{\mathbf{i}_{\mathbf{c}}}$ dépend uniquement des pertes dans l'ensemble de la surface $S$ des parois de la cavité tandis que la surtension $Q_{\mathrm{c}}$ est liée aux pertes dans l'ensemble des parois à l'exclusion de la surface $s$ occupée par l'échantillon.

De la définition du facteur de surtension, on tire :

$$
\frac{Q_{\mathrm{i}_{\mathrm{c}}}}{Q_{\mathrm{c}}}=\frac{\iint_{S-s} H^{2} \mathrm{~d} S}{\iint_{S} H^{2} \mathrm{~d} S}=1-\frac{\iint_{S} H^{2} \mathrm{~d} S}{\iint_{S} H^{2} \mathrm{~d} S}=1-\alpha .
$$

La cavité résonne dans le mode $\mathrm{TE}_{101}$; elle est réalisée à l'aide d'un morceau de guide standard bande $X$; sa longueur est $22,4 \mathrm{~mm}$. L'échantillon occupe une surface de $0,20 \mathrm{~cm}^{2}$.

Le calcul donne : $\alpha=0,025$; ce terme étant petit devant l'unité, nous pouvons écrire : $Q_{\mathrm{c}} \simeq Q_{\mathrm{i}_{\mathrm{c}}}(1+\alpha)$.
ExEMPLE. - Dans le cas de nos expériences avec le composé $\mathrm{NdCo}_{3}$ à la température ambiante, nous avons trouvé : $Q_{\mathrm{i}_{\mathrm{o}}}=4060 ; Q_{\mathrm{i}_{\mathrm{c}}}=5080$ et par suite : $Q_{0} \simeq 18000$.

Annexe 3. Exploitation des résultats de mesure. Chaque relevé de courbe $i_{\mathrm{r}}(H)$ est accompagné de la mesure des facteurs de surtension suivants :

$Q_{\mathrm{i}_{\mathrm{m}}}$ : surtension interne de la cavité avec échantillon au maximum d'absorption (résonance magnétique). Une telle mesure comporte celles de : $i_{1}$. - Courant détecté avec court-circuit substitué à cavité ; ce courant est constant au cours des diverses mesures (puissance hyperfréquence incidente, maintenue constante) ;

$i_{\mathrm{r}_{\mathrm{m}}}$. - Courant détecté dans l'état considéré ;

$v_{\mathrm{m}}$. - Variation de la tension réflecteur du klystron correspondant à $2 \Delta f$ (demipuissance réfléchie).

(Se référer à l'annexe 2.)

$Q_{\mathrm{i}_{0}}$ : surtension interne de la cavité avec échantillon, sous champ élevé $\left(\mu_{\mathrm{R}}=1\right)$. Mesures de $i_{1}, i_{\mathrm{r} 0}, v_{0}$. $Q_{\mathrm{i}_{\mathrm{c}}}$ : surtension de la cavité vide. Mesures de $i_{1}, i_{\mathrm{r}_{\mathrm{c}}}, v_{\mathrm{c}}$.

On a, par ailleurs, déterminé expérimentalement le facteur $\varphi=\Delta f / \Delta V_{\mathrm{r}}$; dans le cas de nos mesures, avec klystron 6975 fonctionnant en mode $6-3 / 4$, on trouve : $\varphi=175 \times 10^{4} \mathrm{~Hz} / \mathrm{V}(f=9,3 \mathrm{GHz})$.

Dans chaque cas, nous calculons le coefficient de réflexion : $|\mathcal{R}|=\sqrt{i_{\mathrm{r}} / i_{1}}$, le coefficient de couplage :

$$
\beta=(1+|\mathcal{R}|) /(1-|\mathcal{R}|) \text {, }
$$

ou :

$$
\beta=(1-|\mathcal{R}|) /(1+|\mathcal{R}|),
$$

puis

$$
Q_{\mathrm{i}}=(1+\beta) \frac{f_{0}}{v \varphi}
$$

et enfin

$$
Q_{\mathrm{e}}=(1 / \beta) Q_{\mathrm{i}} \text {. }
$$

$Q_{\mathrm{e}}$ doit avoir la même valeur dans les divers cas si $\beta$ a été bien défini (sur- ou sous-couplage) ; nous disposons là d'un moyen de contrôle simple.

Le coefficient de couplage de notre cavité, vide, est très légèrement inférieur à l'unité $(0,96)$. De ce fait, la cavité sera toujours sous-couplée. La valeur que l'on attribuera à $Q_{\mathrm{e}}$ sera la moyenne des diverses valeurs, normalement très peu différentes, que l'on aura obtenues pour les divers cas.

Chaque facteur de surtension interne est calculé à l'aide de la relation générale suivante :

$$
Q_{\mathrm{i}}=(1+\beta) \frac{f_{0}}{v \varphi}=\frac{2}{1+|\mathcal{R}|} \frac{f_{0}}{v \varphi}=\frac{2}{1+\sqrt{i_{\mathrm{r}} / i_{1}}} \frac{f_{0}}{v \varphi}
$$

(cas du sous-couplage). 
Calcul de $Q_{0}$. $-Q_{0}$ est déduit des mesures de $Q_{\mathrm{i}_{0}}$, $Q_{\mathrm{i}_{\mathrm{c}}}$ et du facteur correctif $\alpha$ calculé. On a :

$$
1 / Q_{\mathrm{i}_{0}}=\left(1 / Q_{\mathrm{c}}\right)+\left(1 / Q_{0}\right) \text { et } Q_{\mathrm{c}}=(1+\alpha) Q_{\mathrm{i}_{\mathrm{c}}} .
$$

Il en découle :

$$
Q_{0}^{T}=\frac{(1+\alpha) Q_{\mathrm{i}_{\mathrm{c}}} Q_{\mathrm{i}_{0}}}{(1+\alpha) Q_{\mathrm{i}_{\mathrm{c}}}-Q_{\mathrm{i}_{0}}} .
$$

Pour le calcul de $Q_{0}$, comme d'ailleurs pour celui des autres grandeurs à expliciter, on fera intervenir le plus directement possible les résultats des mesures initiales. C'est ainsi que l'on posera :

$$
Q_{0}=2 \frac{f_{0}}{\varphi} \frac{1+\alpha}{(1+\alpha)\left(1+\left|\mathcal{R}_{0}\right|\right) v_{0}-\left(1+\left|\mathcal{R}_{\mathrm{c}}\right|\right)} .
$$

CALCUL DE $\mu_{R_{1}}^{1 / 2}$ PAR APPLICATION DE LA RELATION (9).

$$
\mu_{\mathrm{R}_{1}}^{1 / 2}=1+\left(\frac{1}{\beta_{1}}-\frac{1}{\beta_{0}}\right) \frac{Q_{0}}{Q_{\mathrm{e}}} .
$$

$Q_{\mathrm{e}}$ étant déduit de la mesure de $Q_{\mathrm{i}_{0}}$, on a :

$$
Q_{\mathrm{e}}=\frac{1}{\beta_{0}} Q_{\mathrm{i}_{\mathrm{o}}}=\frac{2}{1-\left|\mathcal{R}_{0}\right|} \frac{f_{0}}{\varphi v_{0}} \text {. }
$$

Par ailleurs :

$$
\frac{1}{\beta_{1}}-\frac{1}{\beta_{0}}=2 \frac{\left|\mathcal{R}_{1}\right|-\left|\mathcal{R}_{0}\right|}{\left(1-\left|\mathcal{R}_{1}\right|\right)\left(1-\left|\mathcal{R}_{0}\right|\right)} .
$$

Portant ces divers résultats dans l'expression (9), on obtient :

$$
\begin{aligned}
\mu_{R_{1}}^{1 / 2}= & 1+2 \frac{\left|\mathcal{R}_{1}\right|-\left|\mathcal{R}_{0}\right|}{1-\left|\mathcal{R}_{1}\right|} \times \\
& \times \frac{(1+\alpha) v_{0}}{(1+\alpha)\left(1+\left|\mathcal{R}_{0}\right|\right) v_{0}-\left(1+\left|\mathcal{R}_{\mathrm{c}}\right|\right) v_{\mathrm{c}}} .
\end{aligned}
$$

$\mathrm{Au}$ cours d'un essai, seul $\mathcal{R}_{1}$ varie avec le champ magnétique appliqué.

Exemple. - Cas du composé $\mathrm{NdCo}_{3}$ à la résonance à la température ambiante ; les résultats expérimentaux ont été les suivants : $\mathcal{R}_{1}=0,204$;

$$
\mathcal{R}_{0}=0,1325 ; \mathcal{R}_{\mathrm{c}}=0,02 ; v_{\mathrm{c}}=2,05 ; v_{0}=2,31 .
$$

L'application de la relation $\left(9^{\prime}\right)$ donne: $\mu_{\mathrm{R}} \simeq 3$ (U. E. M. C. G. S.).

\section{Bibliographie}

[1] Marchand (A.) et Lancia (R.), C. R. Acad. Sci. Paris, 1968, 267, 1323.

[2] Marchand (A.) et Lancia (R.), Colloque International sur les Eléments des Terres Rares, C. N. R. S., Grenoble, 1969.

[3] Lancia (R.), Marchand (A.) et Coumes (A.), $J$. Physique, 1970, 31, 109.

[4] Young (J. A. Jr) et Uehling (E. A.), Phys. Rev. 1954, 94, 544.
[5] Bloembergen (N.), Phys. Rev. 1950, 78, 572.

[6] Van Jleck (J. H.), Phys. Rev. 1950, 78, 266.

[7] Decailliot (M.) et Uebersfeld (J.), C. R. Acad. Sci. Paris, 1966, 262, 141.

[8] Strub (A. S.), J. Phys. Rad. 1959, 20, 52 S.

Dans cet article, lire $: i_{1}^{\prime \prime}=\left(i_{1}^{\prime}+i_{1_{\text {res }}}\right) / 2$ au lieu de $: i_{1}^{\prime \prime}=\left(i_{1}^{\prime}-i_{1_{\text {res }}}\right) / 2$. 